\title{
Fatores prognósticos e impacto da comorbidade na laringectomia fronto-lateral
}

\section{Prognostic factors and comorbidity impact upon the frontolateral laryngectomy}

\author{
Rogério Aparecido Dedivitis, TCBC-SP; Jozías de Andrade-Sobrinho, ECBC-SP²; Mario Augusto Ferrari de Castro³
}

\author{
RE S U M O
}

\begin{abstract}
Objetivo: Avaliar sobrevida, impacto da comorbidade, complicações e fatores de falha da laringectomia como tratamento de tumores malignos glóticos. Métodos: Foram analisadas 38 pacientes com tumor glótico sob estadiamento clínico T1b/T2NOM0 submetidos à laringectomia fronto-lateral com reconstrução, de janeiro de 1995 a dezembro de 2006. Foram avaliados os resultados oncológicos, comorbidades (através da escala Adult Comorbidity Evaluation - 27 ACE-27) e complicações, sendo correlacionados com dados demográficos e características do tumor. Resultados: Oito pacientes apresentaram recidiva local e foram resgatados cirurgicamente. Complicações não foram verificadas em 33 pacientes. Não houve diferença significativa das sobrevidas global em cinco anos e livre de doença ao considerarem-se as diferentes categorias de comorbidades. Somente o envolvimento patológico das margens mostrou diferenças significativas na sobrevida global $(p=0,0033)$ e sobrevida livre de doença $(p<0,0001)$. Conclusão: $A$ sobrevida global em cinco anos foi de $67,6 \%$ e a sobrevida livre de doença de $73,7 \%$; a comorbidade não representou fator prognóstico independente; o índice de complicações pós-operatórias foi de 13,2\% e somente o envolvimento patológico das margens mostrou diferenças significativas na sobrevida global e livre de doença.
\end{abstract}

Descritores: Laringe. Neoplasia laríngeas. Laringectomia. Comorbidade.

\section{INTRODUÇÃO}

$\mathrm{O}$ tratamento conservador para pacientes portadores de carcinoma de células escamosas originário da prega vocal é a radioterapia ou o amplo espectro das laringectomias parciais, também referidas como operações de preservação da laringe. Trata-se de um grupo de procedimentos que remove uma porção da laringe enquanto mantêm função satisfatória de respiração, deglutição e fala sem, no entanto, comprometer os índices de cura e sem a necessidade do traqueostoma definitivo ${ }^{1,2}$.

No protocolo deste estudo, lesões glóticas no estádio clínico T1b, ou seja, com invasão de comissura anterior (e, eventualmente, de um segmento da prega vocal contra-lateral) não são tratadas por via endoscópica ${ }^{3}$. Apesar de o tendão da comissura anterior - onde ocorre a confluência dos ligamentos vocais, pericôndrio interno da cartilagem tireóide e ligamento tíreo-epiglótico - ser considerado região de resistência à disseminação do tumor, os sítios cranial e caudal a esse tendão são áreas de fraqueza e, portanto, propensas a recidiva tumoral ${ }^{4}$.

A abordagem cirúrgica ideal para o tratamento do tumor glótico precoce envolve a extirpação da lesão sob a menor ressecção possível de mucosa, músculos e arcabouço laríngeos. Neel III et al. descreveram, em 182 pacientes selecionados, sobrevida atuarial em cinco anos de $95 \%{ }^{5}$. Em uma série de 72 casos submetidos à laringectomia fronto-lateral com seguimento de cinco a 15 anos, Schwerdtfeger e Gosepath (1990) verificaram sobrevida a três anos de $94,3 \%$ e a cinco anos de $79 \%$. Em uma série, estudada por Fiorella et al., de 150 pacientes submetidos à laringectomia fronto-lateral, 66\% estavam sem evidência de doença após cinco anos ${ }^{7}$. Na experiência de Calzada Uriondo et al., a sobrevida em cinco anos foi de $87,5 \%^{8}$.

A evolução dos pacientes com câncer de laringe pode ser afetada por vários fatores e há vários meios de avaliar o prognóstico. Esses pacientes frequentemente apresentam outras doenças e condições médicas em adição ao câncer, denominadas comorbidades. Elas podem influenciar a opção de tratamento, o índice de complicações e podem ainda confundir a análise da sobrevida. Informação precisa sobre comorbidade juntamente com características demográficas e comportamentais do paciente, sítio e estadiamento do tumor são essenciais para um completo ajuste do risco em câncer ${ }^{9}$. Os procedimentos cirúrgicos de cabeça e pescoço mostram substanciais índices de complicações, pouco se sabendo sobre o impacto de tais fatores sobre os índices de complicações e mortalidade ${ }^{10}$. 
Os objetivos deste trabalho são avaliar, em um grupo de pacientes submetidos à laringectomia fronto-lateral com reconstrução, os seguintes aspectos: sobrevida; impacto da comorbidade; complicações e fatores de falha no tratamento.

\section{MÉTODOS}

Para compor o presente estudo foram analisadas 38 pacientes submetidos à laringectomia parcial vertical fronto-lateral com reconstrução pelo retalho bipediculado do músculo esterno-hióideo com pericôndrio externo da cartilagem tireóidea, no período de janeiro de 1995 a dezembro de 2006. O trabalho foi aprovado pelo Comitê de Ética em Pesquisa da Universidade Metropolitana de Santos sob o número 018/2008. O projeto de pesquisa foi registrado no UMIN Clinical Trials Registry, do Japão, sob o número R000002002.

Foram estudados pacientes portadores de carcinoma de células escamosas de andar glótico da laringe, documentado por exame histopatológico do tumor primário, sob estadiamento clínico T1b / T2NOMO. Todos os pacientes tinham, no mínimo, 12 meses de intervalo pós-operatório. Foram excluídos pacientes que submetidos a qualquer modalidade prévia de tratamento.

A amostra de 38 pacientes incluídos constituiuse de 33 do gênero masculino e cinco do feminino. Todos eram caucasianos. A idade variou de 42 a 85 anos, com média de 62,2 anos. Trinta e um eram tabagistas crônicos importantes, enquanto 26 etilistas crônicos importantes. 0 tempo de história clínica variou de três a 12 meses, sendo a disfonia o sintoma comum a todos. O estadiamento clínico dos pacientes foi o seguinte: 33 T1bNOMO e cinco T2NOMO. Quanto à ressecção de cartilagem aritenóide, não foi realizada em 30 pacientes $(78,9 \%)$, sendo feita aritenoidectomia parcial em três $(7,9 \%)$ e total em cinco $(13,2 \%)$.

O período de internação variou de dois a seis dias, com mediana de três. Um dreno laminar fino foi inserido no tecido subcutâneo, acima dos músculos prétireoidianos e mantido até a manhã seguinte. Nenhum paciente necessitou de alimentação por sonda nasogástrica. A alimentação por via oral iniciou-se no primeiro dia de pós-operatório em 35 pacientes e no segundo dia em três. Dois pacientes evoluíram com estenose glótica, não tendo sido possível a decanulização. Todos os demais puderam ser decanulizados, sendo a retirada da cânula de traqueostomia realizada de sete a 16 dias, com mediana de 10 dias.

Os pacientes foram estudados até a data de sua última consulta ou do óbito. Foram considerados os seguintes desfechos: paciente vivo sem evidência de doença; paciente vivo com doença; óbito pelo câncer; e óbito assintomático (por outras causas que não o câncer). As seguintes causas de óbito foram consideradas: recidiva local; recidiva regional; metástase à distância e comorbidade (outras causas).
As seguintes complicações foram avaliadas: 1) no período perioperatório: hemorragia; infecção de parede; pneumotórax - diagnosticado clinicamente e confirmado por exame radiológico; seroma - baseado na palpação clínica; e enfisema de subcutâneo além da região cervical anterior; 2) dentro do período de um mês de pós-operatório: edema de aritenóide - através do exame de laringoscopia; fístula faringo-cutânea ou laringo-cutânea e pneumonia - com diagnóstico clínico e radiológico; 3) dentro do período de três meses de pós-operatório: disfagia; estenose laríngea - impossibilitando a decanulação do paciente; insuficiência glótica - manifestando-se clínica e laringoscopicamente por aspiração e quelóide.

Para a avaliação da comorbidade, o "tempo zero" foi definido como a data da laringectomia frontolateral, que foi a primeira terapia antineoplásica dirigida contra o câncer da laringe. Informação de cada paciente foi extraída dos prontuários retrospectivamente e, no "tempo zero" , foram inseridos os dados demográficos básicos, tabagismo, etilismo, antecedentes médicos, sintomas e tempo de duração, presença de tumores múltiplos, resultados dos métodos de imagem, achados histopatológicos, descrição do exame locoregional e a classificação TNM. Os dados coletados posteriormente incluíram detalhes da terapia inicial, recidiva tumoral, tempo acompanhamento e status na última avaliação ou até notificação do óbito ${ }^{11}$. Empregou-se a escala Adult Comorbidity Evaluation - 27 (ACE27) ${ }^{12}$, que inclui 27 diferentes elementos de comorbidade compostos por vários sistemas de órgãos (como cardiovascular, respiratório, gastrintestinal, renal, endócrino, neurológico e imunológico, distúrbios em nível psiquiátrico e reumatológico, malignidade prévia ou coexistente, abuso de álcool e peso corporal). A comorbidade é dividida em um sistema de severidade com três categorias, definidas como grave, moderada ou leve. Um escore global de comorbidade é determinado de acordo com o mais elevado escore isolado, exceto quando dois ou mais componentes com Grau 2 estão presentes. Nessa situação, o escore global de comorbidade é designado Grau 3.

Os seguintes aspectos foram avaliados no sentido de encontrar-se correlação com a recidiva local: idade; gênero; índice de comorbidade; tabagismo; etilismo; estadiamento clínico; ressecção de aritenóide; envolvimento patológico das margens e complicações pós-operatórias. Foi considerada falha no tratamento a recidiva tumoral.

A análise estatística deu-se pela distribuição de freqüências e foi utilizada para descrever as variáveis categóricas e as medidas de tendência central e de variabilidade para as numéricas ou contínuas. As probabilidades de sobrevida global e livre de doença foram estimadas pelo método de Kaplan-Meier e a comparação entre as curvas de sobrevida foi verificada através do teste de log rank considerando-se nível de significância de 5\%. O tempo de seguimento considerado foi o intervalo entre a data de realização da operação e a do óbito ou última informação quando sob censura. As sobrevidas para comorbidades foram calculadas agrupando-se de duas maneiras e mostradas nas tabelas de sobrevida. 


\section{RESULTADOS}

Dos 38 pacientes, oito apresentaram recidiva local e foram resgatados cirurgicamente por laringectomia total a campo alargado e radioterapia adjuvante. Após o referido resgate, seis permanecem vivos e sem evidência de doença durante seguimento médio de 40 meses. Os demais 29 pacientes, no momento do estudo, estavam vivos e sem evidência de doença, com acompanhamento que variou de 12 a 75 meses, mediana de 40 meses Tabela 1 e Figuras 1 e 2.

Com relação a complicações, não foram verificadas em 33 pacientes. Quanto às complicações perioperatórias, houve dois casos de infecção de parede $(5,3 \%)$, tratados por antibioticoterapia, não ocorrendo hemorragia, pneumotórax, seroma e enfisema de subcutâneo além da região cervical anterior. Houve um caso de pneumonia dentro de um mês de pós-operatório, tratado por antibioticoterapia, não sendo observadas outras complicações (edema de aritenóide; fístula faringo-cutânea ou laringo-cutânea e pneumonia). Dois pacientes apresentaram, como complicação tardia, estenose laríngea, impossibilitando sua decanulação. Disfagia, insuficiência e quelóide não ocorreram.
A tabela 2 mostra que não houve diferença estatisticamente significativa das sobrevidas global em cinco anos e livre de doença ao considerarem-se as diferentes categorias de comorbidades, baseando-se na escala ACE27.

Quanto à avaliação dos fatores de falha do tratamento, somente o envolvimento patológico das margens mostrou diferenças significativas na sobrevida global ( $p=0,0033)$ e sobrevida livre de doença $(p<0,0001)$, com menor sobrevida para as margens positivas. Faixa etária mostrou-se marginalmente significativa para sobrevida livre de doença, sendo menor a sobrevida para os de faixa etária inferior a 60 anos $(p=0,0515)$ (Tabelas 3 e 4).

\section{DISCUSSÃO}

Lesões limitadas à prega vocal envolvendo a comissura anterior podem ser abordadas pela laringectomia fronto-lateral. Infiltração do ventrículo, da metade caudal da prega vestibular ou do processo vocal da cartilagem aritenóide são também consideradas indicações ${ }^{13}$. Pequeno grau de extensão infraglótica podem ser efetivamente tratados por meio desse procedimento. $\mathrm{O}$ tendão do mús-

Tabela 1 - Distribuição da casuística de acordo com variáveis demográficas, clínicas e de tratamento.

\begin{tabular}{|c|c|c|}
\hline Variável & Categoria / Medidas & Freq (\%) / Medidas \\
\hline \multirow[t]{4}{*}{ Idade (anos) } & Variação & $42-85$ \\
\hline & Mediana & 63 \\
\hline & Média & 62,2 \\
\hline & Desvio Padrão & 10,5 \\
\hline \multirow[t]{2}{*}{ Gênero } & Masculino & $33(86,8)$ \\
\hline & Feminino & $5 \quad(13,2)$ \\
\hline \multirow[t]{2}{*}{ Tabagismo } & Não & $(18,4)$ \\
\hline & $\operatorname{sim}$ & $31 \quad(81,6)$ \\
\hline \multirow[t]{2}{*}{ Etilismo } & Não & $(31,6)$ \\
\hline & $\operatorname{sim}$ & $26 \quad(68,4)$ \\
\hline \multirow[t]{2}{*}{ Estadiamento clínico } & $\mathrm{T} 1 \mathrm{~b}$ & $33 \quad(86,8)$ \\
\hline & $\mathrm{T} 2$ & $5 \quad(13,2)$ \\
\hline \multirow[t]{3}{*}{ Ressecção de aritenóide } & Não & $(78,9)$ \\
\hline & Parcial & $(7,9)$ \\
\hline & Completa & $(13,2)$ \\
\hline \multirow[t]{2}{*}{ Envolvimento patológico das margens } & Negativo & $(7,9)$ \\
\hline & Positivo & $(21,0)$ \\
\hline \multirow[t]{4}{*}{ Complicações pós-operatórias } & Não & $(86,8)$ \\
\hline & Estenose laríngea & $(5,3)$ \\
\hline & Infecção & $(5,3)$ \\
\hline & Pneumonia & $(2,6)$ \\
\hline \multirow[t]{3}{*}{ Status } & Vivo sem doença & $(57,9)$ \\
\hline & Morte por câncer & $(13,2)$ \\
\hline & Morte Assintomática & $11 \quad(28,9)$ \\
\hline \multirow[t]{2}{*}{ Recidiva local } & Não & $(76,3)$ \\
\hline & $\operatorname{sim}$ & $(23,7)$ \\
\hline \multirow{2}{*}{ Recidiva cervical } & Não & $(94,7)$ \\
\hline & $\operatorname{sim}$ & $(5,3)$ \\
\hline
\end{tabular}




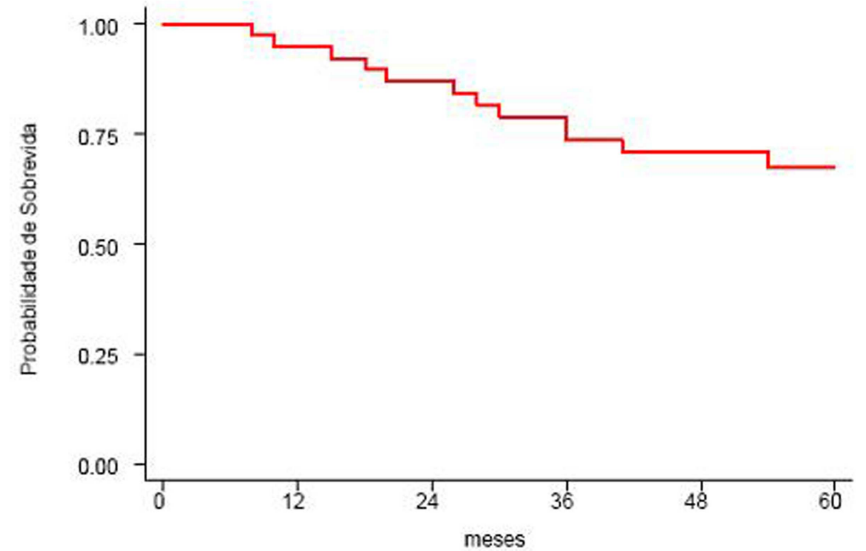

Figura 1 - Sobrevida global (Kaplan-Meier) dos pacientes submetidos à laringectomia fronto-lateral $(n=38)$.

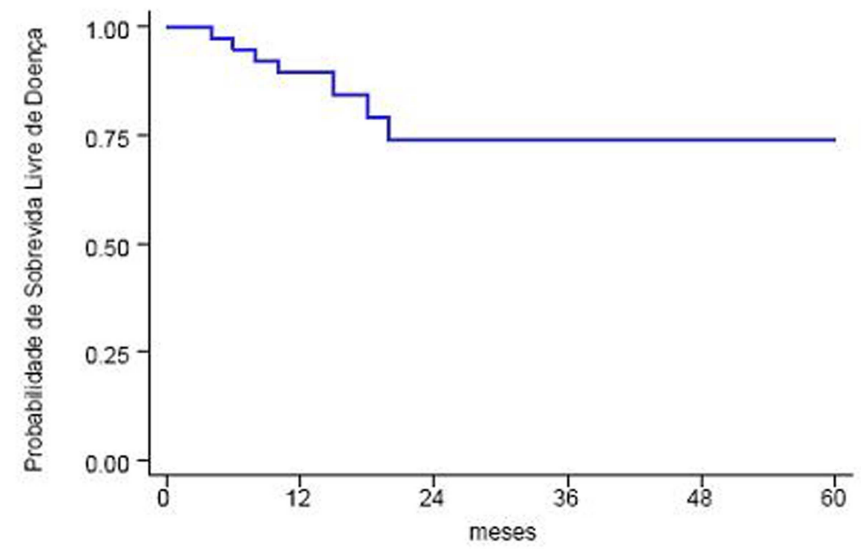

Figura 2 - Sobrevida livre de doença (Kaplan-Meier) dos pacientes submetidos à laringectomia fronto-lateral $(n=38)$.

Tabela 2 - Distribuição das classificações de comorbidades.

\begin{tabular}{lccc}
\hline Classificação das Comorbidades & Categorias & Freq. (\%) & \\
\hline Distribuição das classificações de comorbidades & 0 & $10 \quad(26,3)$ & \\
& 1 & $20 \quad(52,6)$ & \\
Sobrevida global em 5 anos & 2 & $8(21,1)$ & 0,3240 \\
& 0 & 90,0 & \\
Sobrevida livre de doença & 1 & 58,8 & 0,1543 \\
& $>2$ & 60,0 & \\
\end{tabular}

$p$ - valor obtido pelo teste de log rank.

Tabela 3 - Probabilidade de sobrevida global em cinco anos.

\begin{tabular}{|c|c|c|c|}
\hline \multirow[b]{2}{*}{ Variável } & \multicolumn{3}{|c|}{ Sobrevida (\%) } \\
\hline & Categoria & 5-anos & p-valor \\
\hline Sobrevida & global & 67,6 & \\
\hline \multirow[t]{2}{*}{ Faixa etária (anos) } & $<60$ & 54,8 & 0,5680 \\
\hline & $>60$ & 76,4 & \\
\hline \multirow[t]{2}{*}{ Gênero } & Masculino & 62,7 & 0,0989 \\
\hline & Feminino & 100,0 & \\
\hline \multirow[t]{2}{*}{ Tabagismo } & Não & 85,7 & 0,2322 \\
\hline & $\operatorname{sim}$ & 63,4 & \\
\hline \multirow[t]{2}{*}{ Etilismo } & Não & 75,0 & 0,4676 \\
\hline & Sim & 64,4 & \\
\hline \multirow[t]{2}{*}{ Estadiamento clínico } & $\mathrm{T} 1 \mathrm{~b}$ & 71,8 & 0,1417 \\
\hline & $\mathrm{T} 2$ & 40,0 & \\
\hline \multirow[t]{3}{*}{ Ressecção de aritenóide } & Não & 72,3 & 0,3377 \\
\hline & Parcial & 66,7 & \\
\hline & Completa & 40,0 & \\
\hline \multirow[t]{2}{*}{ Envolvimento patológico das margens } & Negativo & 79,1 & 0,0033 \\
\hline & Positivo & 25,0 & \\
\hline \multirow{2}{*}{ Complicações pós-operatórias significativas } & Não & 68,7 & 0,9313 \\
\hline & Sim & 60,0 & \\
\hline
\end{tabular}

$p$ - valor obtido pelo teste de log rank. 
Tabela 4 - Probabilidade de sobrevida livre de doença.

\begin{tabular}{|c|c|c|c|}
\hline \multirow[b]{2}{*}{ Variável } & \multicolumn{3}{|c|}{ Sobrevida (\%) } \\
\hline & Categoria & 5-anos & $p$-valor \\
\hline Sobrevida & Livre de doença & 73,7 & \\
\hline \multirow[t]{2}{*}{ Faixa etária (anos) } & $<60$ & 56,3 & 0,0515 \\
\hline & $>60$ & 86,4 & \\
\hline \multirow[t]{2}{*}{ Gênero } & Masculino & 69,7 & 0,1813 \\
\hline & Feminino & 100,0 & \\
\hline \multirow[t]{2}{*}{ Tabagismo } & Não & 85,7 & 0,4180 \\
\hline & Sim & 71,0 & \\
\hline \multirow[t]{2}{*}{ Etilismo } & Não & 83,3 & 0,3992 \\
\hline & Sim & 69,2 & \\
\hline \multirow[t]{2}{*}{ Estadiamento clínico } & $\mathrm{T} 1 \mathrm{~b}$ & 75,8 & 0,4567 \\
\hline & $\mathrm{T} 2$ & 60,0 & \\
\hline \multirow[t]{3}{*}{ Ressecção de aritenóide } & Não & 73,3 & 0,4964 \\
\hline & Parcial & 100,0 & \\
\hline & Completa & 60,0 & \\
\hline \multirow[t]{2}{*}{ Envolvimento patológico das margens } & Negativo & 86,7 & $<0,0001$ \\
\hline & Positivo & 25,0 & \\
\hline \multirow[t]{2}{*}{ Complicações pós-operatórias significativas } & Não & 72,7 & 0,7755 \\
\hline & Sim & 80,0 & \\
\hline
\end{tabular}

$p$ - valor obtido pelo teste de log rank.

culo vocal insere-se anteriormente diretamente na cartilagem tireóide, área onde não existe pericôndrio interno. Assim, não há barreira de resistência à infiltração tumoral junto à comissura anterior ${ }^{14}$.

Numerosos relatos descrevem técnicas de reconstrução. As vantagens da reabilitação em uma operação em estágio único, associada à laringectomia parcial, são evidentes ${ }^{15}$. O retalho substitui o tecido ressecado, evitando insuficiência glótica, tendo resultados superiores ao não emprego de reconstrução. O retalho bipediculado do músculo esterno-hióideo proporciona resultados fonatórios e função esfincteriana aceitáveis, especialmente em procedimentos unilaterais.

Nesta casuística, houve oito recidivas locais, que puderam ser resgatadas cirurgicamente. Ao avaliarem-se fatores de falha, somente o envolvimento patológico das margens mostrou diferenças significativas nas sobrevidas global e livre de doença, com menor sobrevida para as margens positivas. Margens histológicas negativas não garantem a prevenção da recorrência do tumor. Seguimento pós-operatório rigoroso é a melhor conduta para a detecção precoce de recidiva ${ }^{16}$. Mantilla-Morales et al. (2005) consideram que o controle neoplásico da laringectomia fronto-lateral é similar ao da laringectomia total sempre que as margens cirúrgicas sejam negativas. Advogam a conversão do procedimento para laringectomia total, caso, no trans-operatório, encontre-se invasão tumoral de estruturas extra-glóticas, como sáculo, infraglote e pecíolo da epiglote ${ }^{17}$. A rotina cirúrgica destes autores, após remoção do espécime, é colher fragmentos das margens da ferida do paciente (e não do espécime) e realizar exame histopatológico intra-operatório (congelação), visando a radicalidade da extirpação do tumor. Em todos os casos, o resultado histopatológico final (espécime incluído em parafina) confirmou os achados de margens livres reportados à congelação. Entretanto, em oito casos, foram verificadas margens exíguas no espécime cirúrgico e isso acabou tendo significado estatístico no surgimento de recidiva local.

A razão para melhor evolução em pacientes mais jovens não está clara. Fatores nutricionais podem influenciar a habilidade de resposta do hospedeiro ao tumor. Uma resposta imunológica superior pode ser importante para a reabilitação após o tratamento ${ }^{18}$. O aumento da população idosa e, como consequência, da incidência de doença oncológica permite compreender a interação da idade com as condições de comorbidade e a expectativa de vida. Neste estudo, curiosamente, a faixa etária mostrou-se marginalmente significativa para sobrevida livre de doença, sendo menor a faixa etária inferior a 60 anos.

Doenças coexistentes além do câncer podem afetar fortemente a sobrevida do paciente e também a opção terapêutica. A severidade das condições de comorbidade podem ser graduadas conforme seu impacto na terapêutica adotada e na expectativa de vida. Ao lado dos efeitos diretos na sobrevida, a comorbidade severa pode também ter impacto prognóstico ao alterar a escolha pela modalidade de tratamento. Consequentemente, a presença de comorbidade, além do estádio TNM, pode por vezes determinar a seleção do tratamento e, por conseguinte, a evolução do paciente ${ }^{19}$. Os índices de comorbidade inicialmente identificam as condições e, em seguida, atribuemIhes pesos ou graus de gravidade. Tais pesos baseiam-se no risco relativo de o paciente evoluir a óbito e indicam que as diferentes condições de comorbidade não têm o mesmo impacto ${ }^{20}$. Aplicou-se a escala ACE-27, sem obter 
significado estatístico, talvez pela casuística não ser grande ou ainda pelo fato de não se tratar de opeação oncológica tão longa e agressiva, em pacientes com estadiamento clínico ainda precoce, o que pode sinalizar menor sinergismo entre a doença de base e as comorbidades. Analogamente, o índice de complicações perioperatórias foi baixo e de menor gravidade para os pacientes.
Em conclusão, a sobrevida global em cinco anos foi de $67,6 \%$ e a livre de doença de $73,7 \%$; a comorbidade não representou fator prognóstico independente; o índice de complicações pós-operatórias foi de $13,2 \%$ e somente o envolvimento patológico das margens mostrou diferenças significativas na sobrevida global e livre de doença.

\section{A}

Objective: To evaluate the survival rates, comorbidity impact, complications, and treatment failure facts. Methods: 38 patients clinically staged as T1b / T2NOMO glottic tumors were analyzed. They underwent frontolateral laryngectomy with reconstruction, from January, 1995 to December, 2006. The oncological outcome, comobidity (through the Adult Comorbidity Evaluation -27 ACE27 scale) and complications were studied and correlated to the demographic data and tumor characteristics. Results: Eight patients presented local recurrence being surgically salvaged. Complications were not observed in 33 patients. There was no significant difference on the global and free of disease 5-year survival regarding the diverse comorbidity categories. Only the pathological margins spread of the tumor presented significant difference on the global $(p=0.0033)$ and free of disease survival $(p<0.0001)$. Conclusion: The 5-year global survival was $67.6 \%$ whereas the free of disease survival was $73.7 \%$; the comorbidity did not represent independent prognostic factor; the postoperative complications rate was 13.2\%; and only the pathological margin spread showed significant difference on the global and free of disease survival rates.

Key words: Larynx. Laryngeal Neoplasms. Laryngectomy. Comorbidity.

\section{REFERÊNCIAS}

1. Brumund KT, Gutierrez-Fonseca R, Garcia D, Babin E, Hans S, Laccourreye $\mathrm{O}$. Frontolateral vertical partial laryngectomy without tracheotomy for invasive squamous cell carcinoma of the true vocal cord: a 25-year experience. Ann Otol Rhinol Laryngol. 2005; 114(4):314-22.

2. Dedivitis RA. Resultados na cirurgia conservadora do carcinoma espinocelular de laringe. Rev Bras Cir Cabeça Pescoço. 2008; 37(1):49-50.

3. Dedivitis RA, Guimarães AV, Guirado CR. Outcome after partial frontolateral laryngectomy. Int Surg. 2005; 90(2):113-8.

4. Desloge RB, Zeitels SM. Endolaryngeal microsurgery at the anterior glottal commissure: Controversies and observations. Ann Otol Rhinol Laryngol. 2000; 109(4):385-92.

5. Neel III HB, Devine KD, Desanto LW. Laryngofissure and cordectomy for early cordal carcinoma: outcome in 182 patients. Otolaryngol Head Neck Surg. 1980; 88(1):79-84.

6. Schwerdtfeger FP, Gosepath J. Langzeitstudie nach Frontolateralen Kehlkopfteilresektionen. Laryngorhinootologie. 1990; 69:347-51.

7. Fiorella R, Di Nicola V, Margiatordi F, Fiorella ML. Indications for frontolateral laryngectomy and prognostic factors of failure. Eur Arch Otorhinolaryngol. 1999; 256(8):423-5.

8. Calzada Uriondo G, Sanchez Del Hoyo A, Preciado López JA, Mollá Rudiez FJ, Lacosta Nicolás JL. Laringectomia frontolateral. Nuestra experiencia en La Rioja. Acta Otorrinolaring Esp. 2000; 51(7):6137.

9. Piccirillo JF, Tierney RM, Costas I, Grove L, Spitznagel EL. Prognostic importance of comorbidity in a hospital-based cancer registry. JAMA. 2004; 291(20):2441-7.

10. Ferrier MB, Spuesens EB, Le Cessie S, Baatenburg de Jong RJ. Comorbidity as a major risk factor for mortality and complications in head and neck surgery. Arch Otolaryngol Head Neck Surg. 2005; 131(1):27-32.

11. Castro MAF, Dedivitis RA, Ribeiro KCB. Comorbidity measurement in patients with laryngeal squamous cell carcinoma. ORL J Otorhinolaryngol Relat Spec. 2007; 69(3):146-52. Epub 2007 Jan 30.

12. Paleri V, Wight RG. Applicability of the adult comorbidity evaluation - 27 and the Charlson indexes to assess comorbidity by notes extraction in a cohort of United Kingdom patients with head and neck cancer: A retrospective study. J Laryngol Otol. 2002; 116(3):200-5.

13. Bailey BJ. Partial laryngectomy and laryngoplasty: a technique and review. Trans Am Acad Ophtalmol Otolaryngol. 1966; 70(4):55974

14. Broyles EN. The anterior commossure tendon. Ann Otol. 1943; 52(3):342-5.

15. Conley JJ. Glottic reconstruction and wound rehabilitation. Arch Otolaryngol. $1961 ; 74: 239-42$.

16. Sheen TS, Ko JY, Chang YL. Parcial vertical laryngectomy in the treatment of early glotic cancer. Ann Otol Rhinol Laryngol. 1998; 107(7):593-7.

17. Mantilla-Morales A, Gallegos-Hernández JF, Posada-Sibaja A. Estudio histopatológico de la pieza de laringectomía frontolateral. Puntos de interés clínico. Cir Cir. 2005; 73(4):315-8.

18. Lacy PD, Piccirillo JF, Merritt MG, Zequeira MR. Head and neck squamous cell carcinoma: better to be young. Otolaryngol Head Neck Surg. 2000; 122(2):253-8.

19. Piccirillo JF, Feinstein AR. Clinical symptoms and comorbidity: significance for the prognostic classification of cancer. Cancer. 1996; 77(5):834-42.

20. de Groot V, Beckerman H, Lankhorst GJ, Bouter LM. How to measure comorbidity. a critical review of available methods. J Clin Epidemiol. 2003; 56(3):221-9.

Recebido em 03/12/2008

Aceito para publicação em 03/02/2009

Conflito de interesse: nenhum

Fonte de financiamento: nenhuma

\section{Como citar este artigo:}

Dedivitis RA, Andrade-Sobrinho J, Castro MAF. Fatores prognósticos e impacto da comorbidade na laringectomia fronto-lateral. Rev Col Bras Cir. [periódico na Internet] 2009; 36(5). Disponível em URL: http:// www.scielo.br/rcbc

\section{Endereço para correspondência:}

Rogério Aparecido Dedivitis

E-mail: dedivitis.hns@uol.com.br 